\title{
Computational modeling and experimental analysis for the diagnosis of cell survival/ death for Akt protein
}

\author{
Ayodeji Olalekan Salau ${ }^{{ }^{*}}$ (D) and Shruti Jain ${ }^{2}$ (D)
}

\begin{abstract}
Background: Signalling systems that control cell decisions allow cells to process input signals by apprehending the information of the cell to give one of these two feasible outputs: cell death or cell survival. In this paper, a wellstructured control design methodology supported by a hierarchical design system was developed to examine signalling networks that control cell decisions by considering a combinations of three primary signals (input proteins): the pro survival growth factors, epidermal growth factor (EGF), insulin, and the pro death cytokine, tumour necrosis factor-a (TNF), for AKT/protein kinase B. The AKT actions were examined by using the three input proteins for cell survival/apoptosis for a period of $0-24 \mathrm{~h}$ in 13 different slices for ten different combinations.

Results: Experimental analysis was performed to consider the reactions that were essential to explain the action of AKT. Furthermore, pre-processing and data normalization were performed by using standard deviation, plotting histograms, and scatter plots. Feature extraction and selection were performed using correlation matrix. Radial basis function (RBF) and multiple-layer perceptron (MLP) were used for cell survival/death classification. For all the ten combinations of the three input proteins, 42.85, 347.22, 153.13 were obtained as the minimum value, maximum value, and mean value, respectively, and 126.11 was obtained as the standard deviation for $5-0-5 \mathrm{ng} / \mathrm{ml}$ combinations of TNFEGF-Insulin. The results obtained with MLP 10-8-1 were found to outperform other techniques.

Conclusion: The results from the experimental analysis indicate that it is possible to build self-consistent compendia cell-signalling data based on AKT protein which were simulated computationally to yield important insights for the control of cell survival/death.
\end{abstract}

Keywords: AKT, Apoptosis, Eigenvalues, Protein, Neural network

\section{Background}

Computational biology has recently emerged with a system-level understanding of biological processes. Biological signalling networks process extracellular cues to control the cell divisions such as growth-quiescence, survival/death, and proliferation-differentiation [1]. There are different profound and exciting issues which can be considered. These include robustness of network structures, biological systems and dynamics, and applications

\footnotetext{
* Correspondence: ayodejisalau98@gmail.com

${ }^{1}$ Department of Electrical/Electronics and Computer Engineering, Afe Babalola University, Ado-Ekiti, Nigeria

Full list of author information is available at the end of the article
}

to drug discovery. Bioelectronics is a field of electronics that encompasses a range of biology and electronics topics. One aspect of bioelectronics is processing biological systems in electronic applications (e.g., processing novel electronic components from DNA, nerves, or cells) [2]. It also focuses on physically interfacing electronic devices with biological systems such as cell-electrode, brain-machine, or protein-electrode. Applications in this area include supportive technologies for individuals with brain-related disease or injury, namely: paralysis, artificial retinas, and new technologies for protein structurefunction measurements.

\section{Springer Open}

(c) The Author(s). 2020 Open Access This article is licensed under a Creative Commons Attribution 4.0 International License, which permits use, sharing, adaptation, distribution and reproduction in any medium or format, as long as you give appropriate credit to the original author(s) and the source, provide a link to the Creative Commons licence, and indicate if changes were made. The images or other third party material in this article are included in the article's Creative Commons licence, unless indicated otherwise in a credit line to the material. If material is not included in the article's Creative Commons licence and your intended use is not permitted by statutory regulation or exceeds the permitted use, you will need to obtain permission directly from the copyright holder. To view a copy of this licence, visit http://creativecommons.org/licenses/by/4.0/. 
In recent years, protein kinase small cell inhibitors have been considered as a new area of interest in the diagnosis of cell survival/death [3, 4]. Many such kinases are used by clinicians for the treatment of cancer, chronic inflammatory disease, etc. Cancer can be characterized as a genetic disease [5]. There are three types of genes that are affected: tumour suppressor genes, oncogenes, and stability genes. The classical tumour suppressor genes "RB1" play a major role in controlling cell cycle. Informally, they are present in the retinoblastoma and other tumours. Apoptosis and necrosis are two different forms of cell death. Necrosis is an early disruption of the cell membrane and is associated with organelle swelling, while apoptosis activates the energy required for intracellular interaction which is tightly regulated and conserved throughout evolution. The progressive series of biochemical and morphological changes on cell surfaces of phosphatidylserine, to proteolytic cleavage of numerous intracellular proteins, to nuclear condensation and fragmentation, and the cleavage of DNA into nucleosomal fragments are known as apoptotic cell death [6-8]. One of the important researches in apoptotic cell death is cancer [9]. All the strategies used for killing the tumour/ cancer cells are called anticancer strategies and have been used in clinical oncology, for example, gamma-irradiation, chemotherapy, or immunotherapy. Intrinsic and extrinsic pathways are associated with the stimulation of cell death signal pathways in cancer cells [10].

This paper therefore considered the AKT protein pathways that control cell death/survival decisions using a combination of three input proteins, namely tumour necrosis factor- $\alpha$ (TNF), epidermal growth factor (EGF), and insulin. There are three isoforms of AKT: AKT1, AKT2, and AKT3. For mouse studies, AKT1 is used for cell survival; AKT2 is primarily used for glucose homeostasis, while AKT3 is mainly used for brain development and has a more preponderant role in triple negative tumours. The over-activation of AKT is generated with a myristoylation sequence leading to myrAKT1 or myrAKT2 which maintain the constitutively active protein at the cell membrane. Down-regulation of AKT is generated with shRNA constructions (shAKT1 or shAKT2). The serine $\mathrm{PKB} / \mathrm{AKT} / \mathrm{Rac}$ are the initial identified oncogene and kinase with similar properties as of PKC/PKA. They also play a major role in coordinating the progression of survival, metabolism, and death using the three input proteins (signalling pathways). PI3K/AKT signalling cascade is activated by three signalling pathways through respective tyrosine kinase-like neurotrophin receptors (TrK's). After dimerization, PI3K gives phosphoinositide phosphates (PIP2 and PIP3) at the inner side of the plasma membrane. Phosphoinositidedependent protein kinase 1 (PDK1) later works together with PIP2 and PIP3 to phosphorylate to activate AKT.
In order to perform various functions in the cell, AKT was able to phosphorylate a wide variety of substrate protein. Synthesis peptides with a sequence related to the phosphorylation of GSK3 acts as substrate of AKT kinase activity. AKT is the major factor in different types of cancer. AKT signalling network have diverse downstream effects on cellular metabolism, through either direct regulation of nutrient transporters and metabolic enzymes or the control of transcription factors that regulate the expression of key components of metabolic pathways. It regulates cell growth, survival, and metabolism from exogenous growth stimuli. The molecular events controlling cellular metabolism downstream of PI3K and AKT which represent two major hallmarks of cancer are: growth factor independence through oncogenic signalling and metabolic reprogramming to support cell survival and proliferation. AKT activates NFKB by regulating IKB kinase which results in transcription of pro-survival genes.

This paper examines the cell death/survival decisions for AKT protein pathways. Different parameters were calculated for all the ten different combinations of three inputs proteins. Out of which the best combination was selected using correlation matrix and the results were validated by calculating their Eigenvalues. The selected concentrations were classified using artificial neural networks (ANN). A time series 3D plot was generated for all the best combinations and validated with the training and testing accuracies. The training and testing results yield the same results as that of the neural network $(\mathrm{NN})$ model which accurately predicts cell survival or otherwise cell death. The hallmark of this work is in the description of the predictive model of a cytokine-signal-response compendium used to investigate the regulation of cell fate with the combination of the input proteins for AKT protein.

The rest of this paper is organized as follows. In the "Methods" section, the materials and methods employed for modeling and experimental analysis for the diagnosis of cell survival/death for AKT protein were described in detail. The "Results" section explains the results obtained, and thereafter the results were discussed. This is followed by conclusion and recommendation for future work in the "Conclusion" section.

\section{Methods}

In this section, experimental analysis was performed on HT carcinoma cells. A heat map was obtained from the analysis of ten different concentrations of the three input proteins. Later, features were extracted and selected using a correlation vector. The selected features were classified using different neural network techniques, namely: multiple-layer perceptron (MLP) and radial based function (RBF). The block diagram of the proposed methodology is shown in Fig. 1. The prediction model for cell death/ 


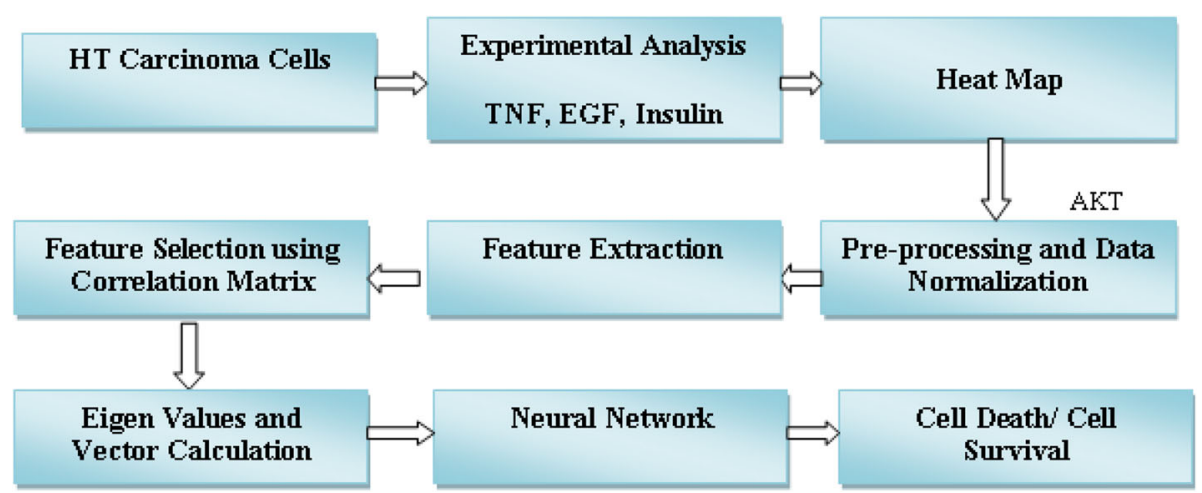

Fig. 1 Proposed methodology for cell death/survival decision for AKT protein

survival was implemented with the proposed method using Statistica Software. In total, we obtained 300 values for each combination of input proteins.

Studies of signalling pathways are focused on depicting downstream and upstream interactions, and then systemizing these interactions into linear cascades that balance information from cell surface receptors to cellular effectors. A bottom-up approach was used for the hierarchical model as shown in Fig. 2.

The bottom-up hierarchical approach starts with the proteins/genes as biological components analogous to the physical layer which consists of active and passive components from electronic elements. In the hierarchy, the next layer is the device layer which comprises of biochemical reactions that regulate the flow of information and manipulate physical processes. The biochemical reactions are equivalent to logic gates which perform computations in a computer. At the module layer, a synthetic biologist could use quiet a number of biological devices to assemble complex pathways that function like integrated circuits. The connection of these modules to each other and their integration into host cells allows the synthetic biologist to extend or modify the behaviour of cells in a programmatic fashion.

HT carcinoma cells are considered for the analysis of cell survival/death by using AKT as a marker protein.

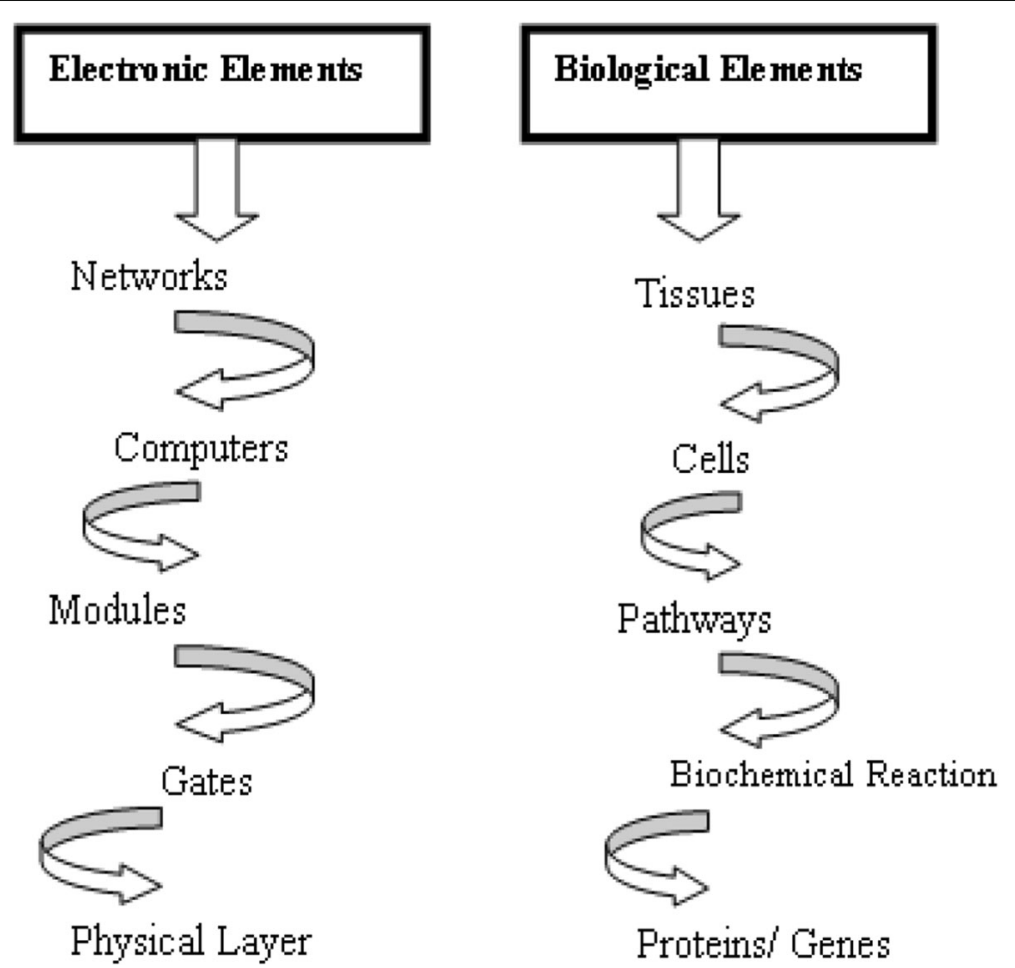

Fig. 2 Proposed hierarchical model of electronic and biological elements 
The experimental analysis was performed by considering different concentrations of TNF such as $0,0.2,5$, and $100 \mathrm{ng} / \mathrm{ml}$. Similarly, different concentrations of EGF, such as 0,1 , and $100 \mathrm{ng} / \mathrm{ml}$, and insulin, such as $0,1,5$, and $500 \mathrm{ng} / \mathrm{ml}$, for making different cultures were analysed for a period of $24 \mathrm{~h}$ by adding $1 / 20$ of diluted stimulus. The $0-24 \mathrm{~h}$ time frame was divided into 0,5 , $15,30,60$, and $90 \mathrm{~min}$, and 2, 4, 8, 12, 16, 20, and $24 \mathrm{~h}$. The cells were exposed to ten cytokine treatments so as to explore systematically the relationship between activation of intracellular signalling cascaded as cytokine receptor interaction and survival death cell fate decisions. All the observations were monitored for a period of 48 h. To explore systematic relationships between the activation of intracellular signalling cascades, cytokine receptor interaction, and apoptosis-survival cell fate decisions cells were exposed to a set of ten different treatments of input proteins. At the 13 time point after cytokine addition, three replicate dishes of cells were harvested to measure kinase activities. Altogether, ten distinct protein signals were examined, namely, (a) assayed in vitro using microtiter-based immunocomplex kinase activity assays: ERK, JNK1, AKT, MK2, and IKK; (b) antibody arrays: phospho-to-total (pt) and phospho total measures of EGFR and AKT; and (c) immunoblotting: five phosphorylation sites on four proteins. Out of the different proteins, AKT signals were examined. Each protein signal was integrated by 12-h, 24-h, and 48-h time frame and then analysed with a set of three input protein treatment. This analysis generated a heat map in which the positions of ten protein signals were defined in comparison to the TNF, EGF, and insulin stimuli. The heat map was prepared for the marker protein of ten different concentrations of input proteins. The ten different concentrations of input proteins (TNFEGF-insulin) are: 0-0-0, 5-0-0, 100-0-0, 0-100-0, 5-1-0, 100-100-0, 0-0-500, 0.2-0-1, 5-0-5, and 100-0-500. Histograms, standard deviation, and scatter plots were calculated to pre-process the data. Different features like mean, maximum, minimum, and standard deviation for training, testing, validation, and overall data were calculated for all ten different concentrations of the input combinations. Correlation vectors were calculated as feature selection techniques and were used to select the best concentrations of input combinations [11]. The results were validated using Eigenvalues and vector calculations. With the help of Eigenvectors, linear transformation is easy to understand. An eigenvector $v$ of a matrix $A$ is independent of the linear transformation: $A v$ $=\lambda v \Rightarrow \lambda(\mathrm{B} u)=\mathrm{A}(\mathrm{B} u)$. Eigenvectors are a set of basic functions that help in describing data variability. The Eigenvalues of our data were calculated from the best combinations of three input proteins which were used to classify cell death/survival for AKT protein. For classification of the proteins, we have employed artificial neural network (ANN) techniques such as MLP and RBF for cell death and cell survival decisions. ANN is a special nonlinear model for classification, clustering as well as regression. There are at least three layers of nodes for a MLP, namely: input layer, hidden layer, and output layer. The input layer consist of input variables which are numeric. Non-numeric data is converted to numeric before it can be used in an ANN technique. This layer is sometimes called the visible layer. The hidden layers consist of layers of nodes between the input and output layers; there may be one or more of these layers. The output layer is a layer of nodes which produce the output variable. Our proposed ANN model for the detection of cell survival/death for AKT is shown in Fig. 3.

ANN techniques are fast becoming a useful approach for signal-processing technologies. In engineering, neural networks serve two important functions: as nonlinear adaptive filters and as pattern classifiers. They are most often adaptive nonlinear systems that learn to perform a function (an input/output map) from data. Adaptive implies that the system parameters change during operation, normally called the training phase. After the training phase, the ANN parameters are fixed and can be deployed to solve problems.

\section{Results}

The experimental observation of cell death/survival from cells treated with ten cytokine combinations of TNF, EGF, and insulin by using AKT was presented in this section. AKT proteins form signalling networks which lead to cell survival/death as shown in Fig. 4 [12].

Futhermore, a similar experimental analysis was carried out as performed in [13, 14]. The results obtained show high similarity. The experimental analysis shows that it is possible to build self-consistent compendia cell-signalling data based on AKT protein which were simulated computationally to yield important insights into the control of cell survival/death. For the purpose of analysis, different experiments were performed with ten different concentrations of three input proteins for $0-24 \mathrm{~h}$ in 13 different slices of AKT protein. The novelty of this work lies in the threefold marker protein selection technique; the first stage includes pre-processing techniques, followed by extraction of different features like minimum, maximum, mean, and standard deviation values to select the best combinations of TNF-EGF-Insulin, and lastly, detection was performed using ANN in the third stage to provide a high detection accuracy and low complexity. The proposed method when tested on AKT protein shows that the MLP provides better results with the least run-time complexity for cell survival/death detection. Since ANN techniques are adaptive to complex 


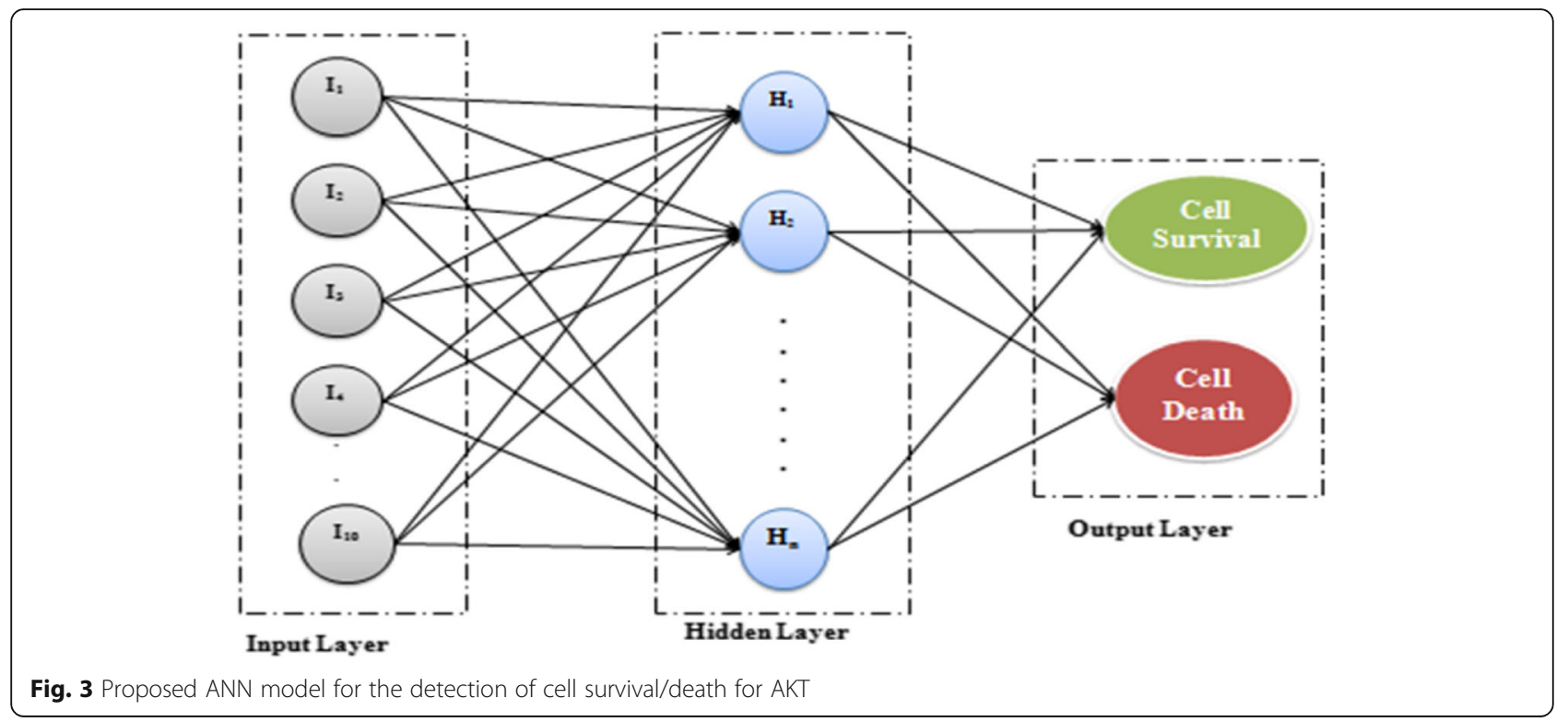

problems, by changing the networks topology, they are able to handle different levels of complexity and predict the desired output of a system when adequate experimental data is provided. One of the advantages of ANNs is it allows the modeling of physical phenomena in complex systems without requiring exhaustive experiments or without requiring explicit mathematical representations.

A nonlinear ANN was employed in this study to uncover important aspects of biological cue-signal-response systems using TNF-, EGF-, and insulin-mediated response of HT-29 human colon carcinoma cells. Although several analyses were performed, the hallmark of this work is in the description of the predictive model of a cytokinesignal-response compendium used to investigate the regulation of cell fate with the combination of the input proteins for AKT protein. The compendium contains more than 10,000 biochemical measurements based on the states and activities of cell-signalling proteins and apoptotic responses in human cells. Experimental databases are common in genomics, majorly because sequence data are structured and homogeneous, with clear start and finish points, and the ease to fuse data. In contrast, cellsignalling data are unstructured and heterogeneous and depend on biological content.

\section{Discussion}

After analysis, four output cellular responses (phosphatidylserine exposure (PE), membrane permeability (MP), nuclear fragmentation (NF), and caspase substrate cleavage $(\mathrm{CC})$ ) were obtained and used to predict cell death/ survival with the consideration of three input proteins

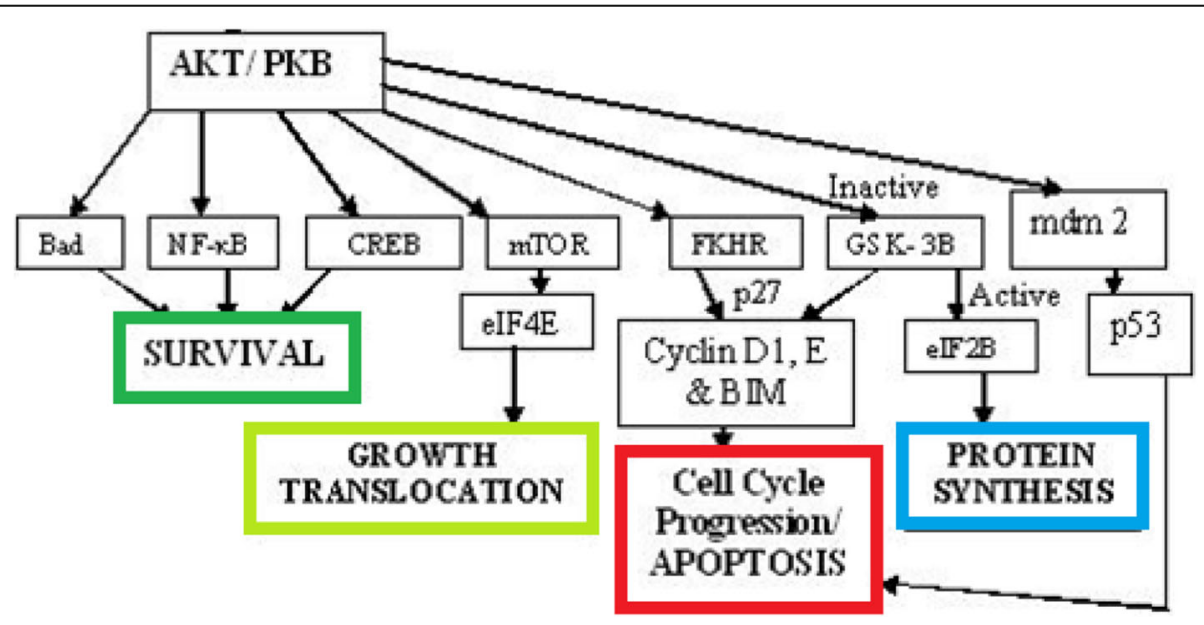

Fig. 4 Pathway for cell survival/death for AKT 


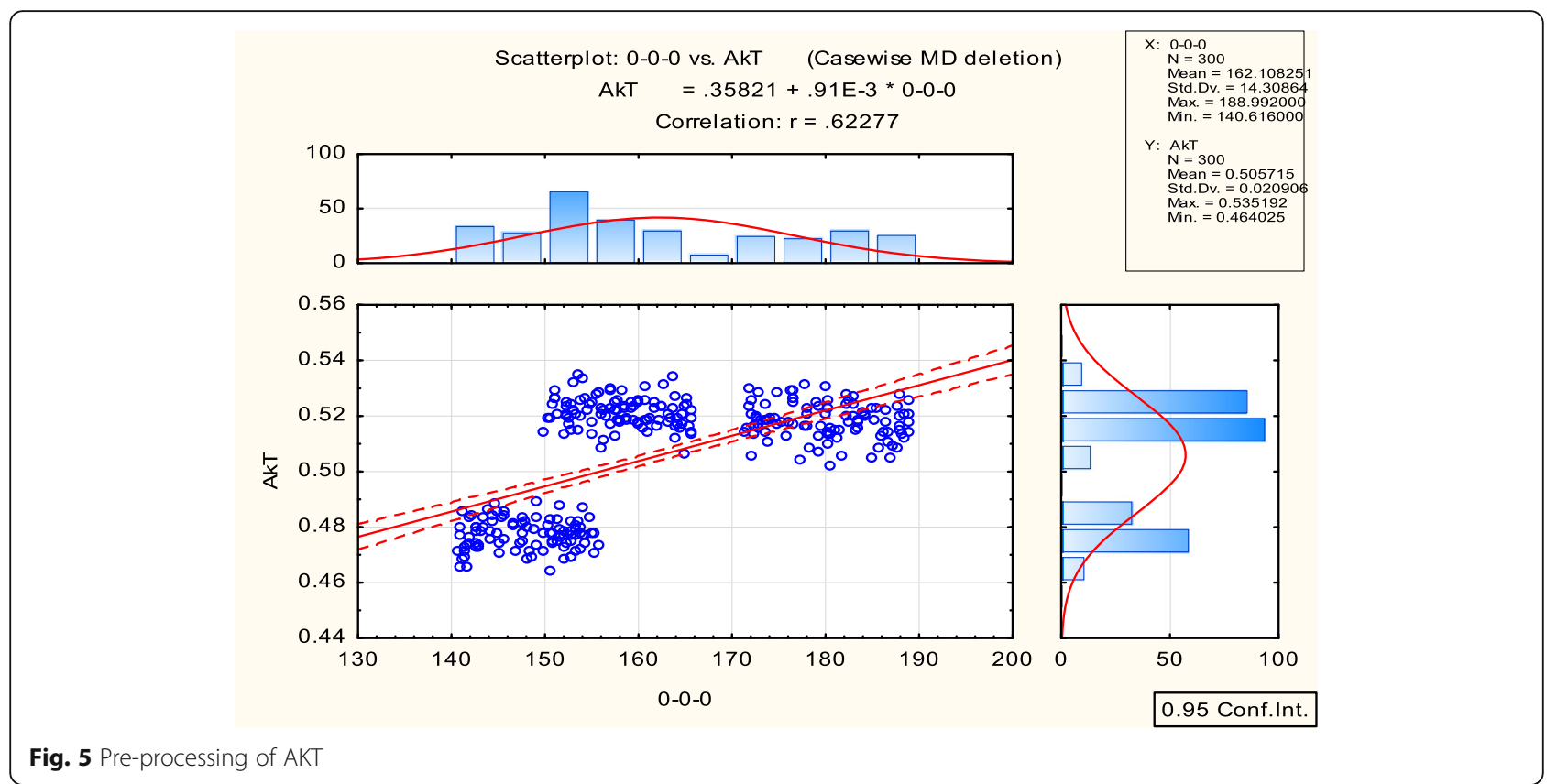

(TNF, EGF, and insulin) using a system biology approach (hierarchical model). Ten different concentrations of three inputs and an average of four outputs were analysed and then normalized giving a final result of 10 inputs and 1 output. Furthermore, a heat map in form of an image was prepared and pre-processed by plotting histograms and scatter plots as shown in Fig. 5. The features like mean, maximum, minimum, and standard deviation for training, testing, validation, and overall data were calculated for the ten different concentrations which are presented in Table 1.

Table 1 shows the mean training, mean testing, mean validation, mean overall data, maximum training, maximum testing, maximum validation, maximum overall data, minimum training, minimum testing, minimum validation, minimum overall data, standard deviation training, standard deviation testing, standard deviation validation, and standard deviation overall data for all the ten different

Table 1 Various extracted features for AKT proteins

\begin{tabular}{|c|c|c|c|c|c|c|c|c|c|c|c|}
\hline \multirow[t]{2}{*}{ Samples } & \multicolumn{11}{|c|}{ Data statistics (AKT data) } \\
\hline & $0-0-0$ & $5-0-0$ & $100-0-0$ & $0-100-0$ & $5-1-0$ & $100-100-0$ & $0-0-500$ & $0.2-0-1$ & $5-0-5$ & $100-0-500$ & AKT \\
\hline Minimum (train) & 140.62 & 164.91 & 83.77 & 161.62 & 81.72 & 151.16 & 140.69 & 143.92 & 42.85 & 110.21 & 0.47 \\
\hline Maximum (train) & 188.99 & 188.99 & 155.71 & 205.55 & 156.59 & 178.01 & 217.53 & 200.24 & 346.22 & 323.42 & 0.54 \\
\hline Mean (train) & 161.66 & 177.72 & 108.54 & 182.22 & 122.48 & 165.65 & 176.99 & 173.12 & 160.28 & 221.72 & 0.51 \\
\hline Standard deviation (train) & 13.77 & 6.03 & 26.16 & 12.19 & 28.25 & 6.73 & 24.66 & 18.02 & 128.77 & 80.89 & 0.02 \\
\hline Minimum (test) & 140.79 & 166.35 & 83.79 & 162.51 & 82.19 & 151.19 & 141.25 & 143.80 & 42.98 & 110.72 & 0.47 \\
\hline Maximum (test) & 188.78 & 188.91 & 154.21 & 205.73 & 154.65 & 178.10 & 215.79 & 198.38 & 347.22 & 323.40 & 0.53 \\
\hline Mean (test) & 162.04 & 176.50 & 108.68 & 182.80 & 119.59 & 166.54 & 177.30 & 170.44 & 171.75 & 230.53 & 0.51 \\
\hline Standard deviation (test) & 14.74 & 6.22 & 27.11 & 11.85 & 28.08 & 7.24 & 24.91 & 18.61 & 132.39 & 80.82 & 0.02 \\
\hline Minimum (validation) & 141.43 & 165.88 & 84.12 & 161.86 & 83.01 & 151.83 & 142.93 & 143.87 & 42.98 & 110.81 & 0.46 \\
\hline Maximum (validation) & 188.35 & 188.74 & 155.17 & 203.85 & 153.44 & 176.94 & 211.48 & 199.64 & 336.94 & 315.43 & 0.53 \\
\hline Mean (validation) & 164.25 & 179.94 & 119.00 & 179.56 & 134.49 & 164.93 & 166.15 & 180.60 & 101.12 & 195.40 & 0.50 \\
\hline Standard deviation (validation) & 18.16 & 4.80 & 28.95 & 5.78 & 4.96 & 6.67 & 18.30 & 5.65 & 16.63 & 38.47 & 0.02 \\
\hline Minimum (overall) & 140.62 & 164.91 & 83.77 & 161.62 & 81.72 & 151.16 & 140.69 & 143.80 & 42.85 & 110.21 & 0.46 \\
\hline Maximum (overall) & 188.99 & 188.99 & 155.71 & 205.73 & 156.59 & 178.10 & 217.53 & 200.24 & 347.22 & 323.42 & 0.54 \\
\hline Mean (overall) & 162.11 & 177.87 & 110.13 & 181.91 & 123.84 & 165.68 & 175.41 & 173.84 & 153.13 & 219.09 & 0.51 \\
\hline Standard deviation (overall) & 14.31 & 6.15 & 26.88 & 11.84 & 27.40 & 6.74 & 24.24 & 17.86 & 126.11 & 79.95 & 0.02 \\
\hline
\end{tabular}


Table 2 Correlation matrix for AKT protein

\begin{tabular}{|c|c|c|c|c|c|c|c|c|c|c|c|c|}
\hline \multirow[t]{2}{*}{ Variable } & \multicolumn{12}{|c|}{$P<0.050, N=300$} \\
\hline & Means & Std. Dev. & $0-0-0$ & $5-0-0$ & $100-0-0$ & $0-100-0$ & $5-1-0$ & $100-100-0$ & $0-0-500$ & $0.2-0-1$ & $5-0-5$ & $100-0-500$ \\
\hline $0-0-0$ & 162.11 & 14.31 & 1.00 & 0.09 & 0.85 & 0.14 & 0.04 & 0.57 & -0.53 & -0.02 & -0.07 & 0.40 \\
\hline $5-0-0$ & 177.87 & 6.15 & 0.09 & 1.00 & 0.31 & -0.49 & 0.53 & -0.16 & -0.47 & 0.50 & -0.53 & -0.44 \\
\hline $100-0-0$ & 110.13 & 26.88 & 0.85 & 0.31 & 1.00 & -0.23 & 0.45 & 0.38 & -0.84 & 0.37 & -0.48 & 0.01 \\
\hline $0-100-0$ & 181.91 & 11.84 & 0.14 & -0.49 & -0.23 & 1.00 & -0.87 & 0.45 & 0.62 & -0.84 & 0.87 & 0.86 \\
\hline $5-1-0$ & 123.84 & 27.40 & 0.04 & 0.53 & 0.45 & -0.87 & 1.00 & -0.37 & -0.81 & 0.95 & -0.99 & -0.87 \\
\hline $100-100-0$ & 165.68 & 6.74 & 0.57 & -0.16 & 0.38 & 0.45 & -0.37 & 1.00 & -0.03 & -0.38 & 0.35 & 0.61 \\
\hline $0-0-500$ & 175.41 & 24.24 & -0.53 & -0.47 & -0.84 & 0.62 & -0.81 & -0.03 & 1.00 & -0.74 & 0.83 & 0.48 \\
\hline $0.2-0-1$ & 173.84 & 17.86 & -0.02 & 0.50 & 0.37 & -0.84 & 0.95 & -0.38 & -0.74 & 1.00 & -0.95 & -0.87 \\
\hline $5-0-5$ & 153.13 & 126.11 & -0.07 & -0.53 & -0.48 & 0.87 & -0.99 & 0.35 & 0.83 & -0.95 & 1.00 & 0.87 \\
\hline $100-0-500$ & 219.09 & 79.95 & 0.40 & -0.44 & 0.01 & 0.86 & -0.87 & 0.61 & 0.48 & -0.87 & 0.87 & 1.00 \\
\hline
\end{tabular}

combinations of three input proteins for AKT protein. For all the ten concentrations of the three input proteins, $42.85,347.22$, 153.13 were obtained as the minimum value, maximum value, and mean value, respectively, and 126.11 as the standard deviation for $5-0-5 \mathrm{ng} / \mathrm{ml}$ combinations of TNF-EGF-Insulin. In addition, the best combinations were selected by using the correlation matrix as presented in Table 2. This matrix is used to describe the dependency between different data sets. In cases of analysis dealing with numerous secondary variables, the correlation matrix is used to describe this dependency effectively.

From Table 2, it is observed that the 0-0-500 concentration is less correlated with each other. Therefore, this concentration can be neglected for the classification of the cells. To validate the results, Eigenvalues of the correlation matrix were also calculated as shown in Fig. 6.
Eigenvalues are a measure of the data variance which are used to reduce the dimension of large datasets by selecting only a few modes with significant values and are also used to find new variables that are uncorrelated. The selected concentrations were used for the classification of the cells using ANN.

An ANN model was developed for the prediction of cell survival/cell death considering ten different combinations. The authors have implemented the propsed neural network model using STATISTICA 2016 data miner software. The proposed ANN model was developed for the prediction of cell survival/cell death considering ten different concentrations of three input proteins. The NN consists of one input layer with ten nodes, where each node corresponds to the different concentrations. It consists of one hidden layer with different hidden nodes and one

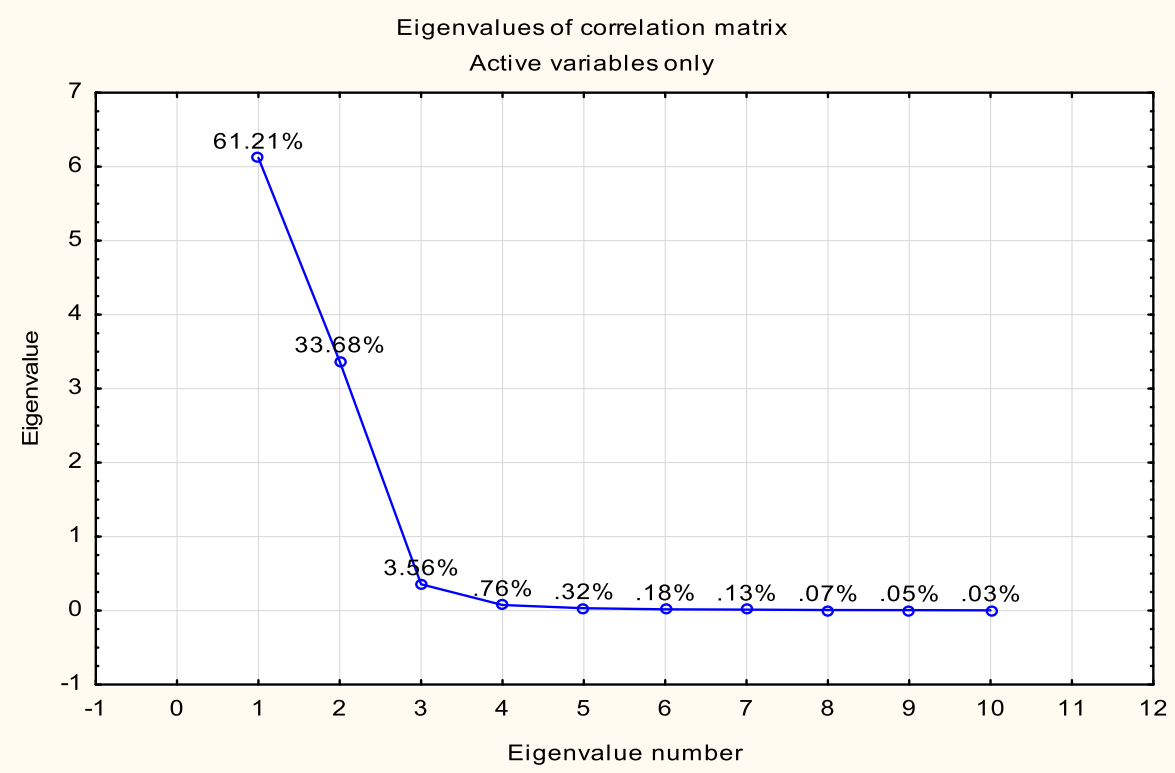

Fig. 6 Plot of Eigenvalues for the correlation matrix 


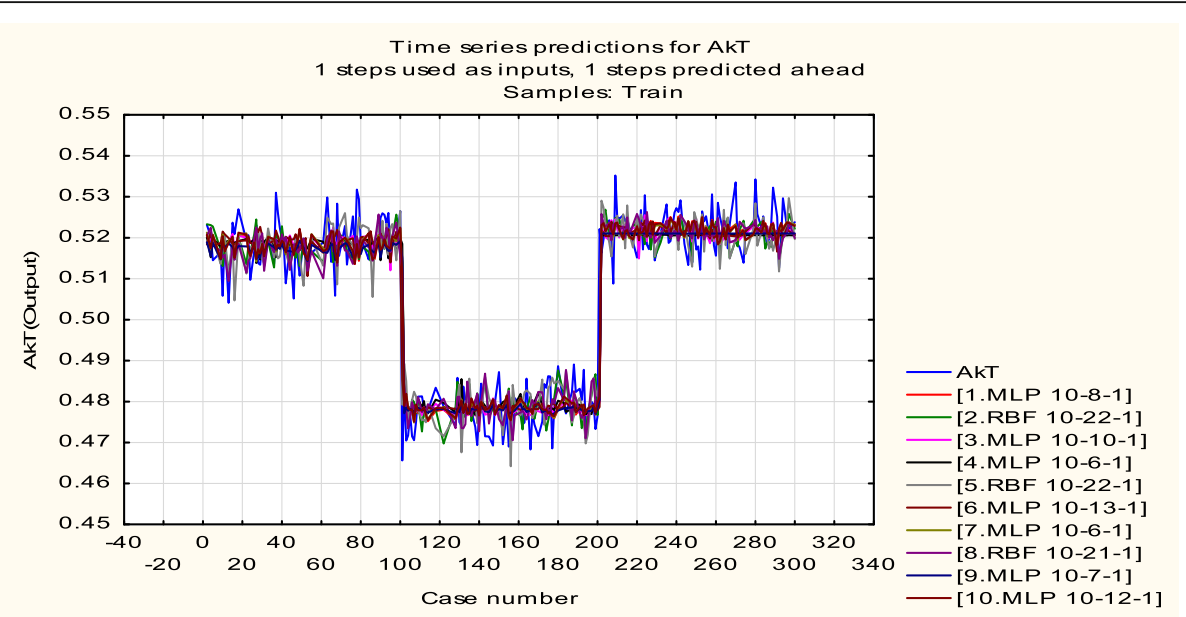

Fig. 7 Time series plot using MLP and RBF techniques to obtain the best 10 results. Note: 10 signifies ten different combinations of three different input proteins, 8 hidden layers, and 1 output cell survival/death result

output layer with 2 nodes. When the predicted output in the second neural network is $>0.5$ it will lead to cell survival; otherwise, it leads to cell death.

In comparison with the statistical analysis, ANN is a nonlinear model which is easy to use and understand and is mostly used for solving various classification and forecasting problems. The results reveal that the proposed ANN model is most adequate to estimate the physiological functions from intracellular protein expressions. Figure 7 shows the time series plot of the 10 results obtained from the analysis using MLP and RBF techniques. A time series graph or plot is a graphical representation of time series data on the $x$-axis (time increments/cases) and on the $y$-axis, the corresponding measured values are plotted. Time series plots are very useful as they illustrate how the values of the measured variable changes over time. In Fig. 7 , the $x$-axis defines the different 300 cases which were considered and the $y$ axis defines the AKT values. Figure 7 further shows the ten different combinations of MLP and RBF. MLP 1012-1 indicates the input-hidden layer-output.

Out of the ten results, MLP 10-8-1 (10 signifies ten different combinations of three different input proteins, 8 hidden layers, and 1 output cell survival/death result) outperforms all other results. The three-dimensional plot for MLP 10-8-1 is shown in Fig. 8 for residual, target, and output. Different threshold values were considered resulting in cases of cell survival/death. Figure 8

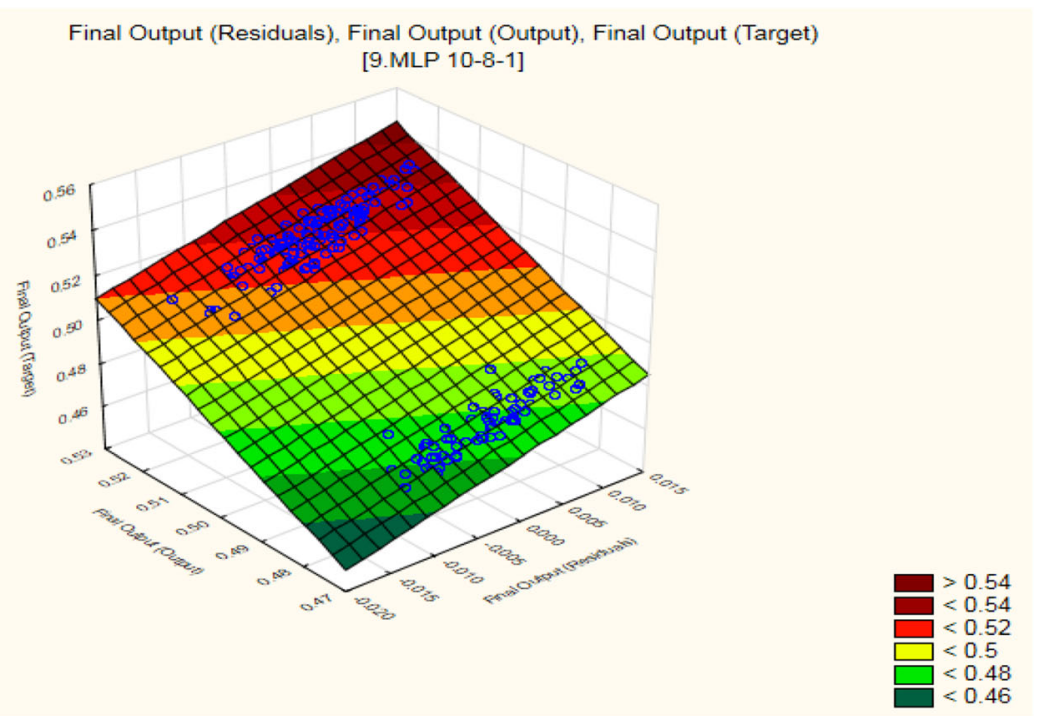

Fig. 8 3D plot of MLP 10-8-1 for final output of residual, target, and output 
Table 3 Training and testing accuracies using different ANN models for AKT

\begin{tabular}{llll}
\hline S/no. & Network name & Training \% accuracy & Test \% accuracy \\
\hline 1 & MLP 10-7-1 & 99.14 & 98.94 \\
2 & RBF 10-22-1 & 99.76 & 99.67 \\
3 & MLP 10-10-1 & 99.77 & 99.76 \\
4 & MLP 10-6-1 & 99.20 & 99.22 \\
5 & RBF 10-22-1 & 99.58 & 99.30 \\
6 & MLP 10-13-1 & 98.48 & 98.43 \\
7 & MLP 10-6-1 & 94.47 & 90.58 \\
8 & RBF 10-21-1 & 99.70 & 99.67 \\
$\mathbf{9}$ & MLP 10-8-1 & $\mathbf{9 9 . 8 9}$ & $\mathbf{9 9 . 8 9}$ \\
10 & MLP 10-12-1 & 98.33 & 98.12 \\
\hline
\end{tabular}

also shows the threshold values which are expressed with different colours.

The results were also validated by calculating the training and testing perfections. Table 3 shows the training and testing accuracy of ten different neural networks using MLP and RBF. The table shows that MLP 10-8-1 gives $99.89 \%$ for both the training and test accuracies

Results obtained in Table 3 validate the values obtained after generating the time series plots as shown in Fig. 7. In addition, the training and testing accuracies yields the same results with the neural network model which accurately predicts cell survival or otherwise cell death. In Table 4, we present a comparison of the results of the proposed method with existing works. The results show that the proposed method outperforms the existing methods for the analysis and determination of cell sur$\mathrm{vival} /$ death.

\section{Conclusion}

Biological systems can create complex structures from very simple systems. In this paper, a series of experimental analysis were performed with ten different concentrations of three input proteins for a period of $0-24 \mathrm{~h}$ in 13 different slices. Based on the experimental analysis, a

Table 4 Comparison of results of research papers with the proposed technique

\begin{tabular}{llll}
\hline S. No. & Technique & Proteins & Accuracy (\%) \\
\hline 1. & GLCM + k-NN [15] & EGFR, IRS, ERK, & 75.60 \\
& & MK2, JNK, FKHR & \\
2. & GLDS + k-NN [6] & AKT & 76.90 \\
3. & DWT (Bior 4.4) + SSVM [7] & ERK, MK2, JNK & 80.00 \\
4. & GLDS + SVM [6] & AKT & 84.60 \\
5. & GLCM + SVM [15] & EGFR, IRS, ERK, & 85.80 \\
& & MK2, JNK, FKHR & \\
6. & $\begin{array}{l}\text { Proposed technique } \\
\text { (MLP ANN) }\end{array}$ & AKT & 99.89 \\
\hline
\end{tabular}

heat map (in the form of an image) was generated for different marker proteins. Initially, the data was preprocessed, and subsequently different features were extracted and selected based on the correlation matrix method. The selected features were then validated by calculating their Eigenvalues. Furthermore, RBF and MLP techniques were applied for the cell death/cell survival decisions. A time series $3 \mathrm{D}$ plot was generated for all the best combinations and validated with its testing accuracy. In the future, different optimization techniques will be applied for the selection of features.

\section{Abbreviations \\ ANN: Artificial neural networks; CC: Caspase substrate cleavage; \\ DNA: Deoxyribo nucleic acid; EGF: Epidermal growth factor; ERK: Extracellular signal regulated protein kinase; GSK3: Glycogen synthase kinase 3; JNK1: c- \\ Jun NH2 terminal kinase 1; MK2: Mitogen-activated protein kinase-activated protein kinase 2; MLP: Multiple-layer perceptron; MP: Membrane permeability; NF: Nuclear fragmentation; NFkB: Nuclear factor kappa B; NN: Neural network; PE: Phosphatidylserine exposure; PDK1: Protein kinase 1; PIP: Phosphoinositide phosphates_-plasma membrane intrinsic protein. The PIP subfamily is subdivided into two groups: PIP1 and PIP2; PKA: Protein kinase A; PKB: Protein kinase B/AKT protein kinase; PKC: Protein kinase C; Rac: Ras-related C3 botulinum toxin substrate; RBF: Radial based function; TNF: Tumour necrosis factor}

\section{Acknowledgements}

Not applicable

Compliance with journal polices

The authors declare that they have abided by all journal polices.

Details of any previous or concurrent submissions

This paper has not been submitted anywhere else for review or has not been published previously.

\section{Authors' contributions}

Both AOS and SJ contributed equally to this paper. AOS formulated the idea, carried out simulations, and wrote some parts of the paper, while SJ provided the method, wrote the results and discussion sections, and participated in writing other parts of the paper. All authors have read and approved the manuscript.

\section{Funding}

The authors declare no funding for this research.

Availability of data and materials

All data generated or analysed during this study are included in this published article.

Ethics approval and consent to participate

Not applicable

Consent for publication

The authors agree to the publication of this paper

\section{Competing interests}

The authors declare that they have no competing interest.

\section{Author details}

'Department of Electrical/Electronics and Computer Engineering, Afe Babalola University, Ado-Ekiti, Nigeria. ${ }^{2}$ Department of Electrical and Communications Engineering, Jaypee University of Information Technology, Solan, Himachal Pradesh, India. 
Received: 6 January 2020 Accepted: 24 March 2020

Published online: 21 April 2020

\section{References}

1. Zhang X, Tang N, Hadden TJ, Rishi AK (2011) AKT, FoxO and regulation of apoptosis. Biochimica et Biophysica Acta 1813:1978-1986

2. Jain S (2018) System modeling of AKT using linear and robust regression analysis. Curr Trend Biotechnol Pharm 12(2):177-186

3. Portt L, Norman G, Clapp C, Greenwood M, Greenwood MT (2011) Antiapoptosis and cell survival: A review. Biochimica et Biophysica Acta 1813: 238-259

4. Schenck A, Goto-Silva L, Collinet C, Rhinn M, Giner A, Habermann B, Brand M, Zerial M (2008) The endosomal protein appl1 mediates AKT substrate specificity and cell survival in vertebrate development. Cell 133:486-497

5. Karege F, Perroud N, Burkhardt S, Schwald M, Ballmann E, La Harpe R, Malafosse A (2007) Alteration in kinase activity but not in protein levels of protein kinase B and glycogen synthase kinase-3beta in ventral prefrontal cortex of depressed suicide victims. Biol Psychiatry 61(2):240-245

6. Jain S, Salau AO (2019) An image feature selection approach for dimensionality reduction based on kNN and SVM for AKT proteins. Cogent Engineering 6(1):1-14. https://doi.org/10.1080/23311916.2019.1599537

7. Jain S (2018) Classification of protein kinase b using discrete wavelet transform. Int J Inform Technol 10(2):211-216. https://doi.org/10.1007/ s41870-018-0090-7

8. De Bruin EC, Medema JP (2008) Apoptosis and non-apoptotic deaths in cancer development and treatment response. Cancer Treat Rev 34:737-749

9. Brunet A, Bonni A, Zigmond MJ, Lin MZ, Juo P, Hu LS, Anderson MJ, Arden KC, Blenis J, Greenberg ME (1999) AKT promotes cell survival by phosphorylating and inhibiting a fork head transcription factor. Cell:857-868

10. Manning BD, Cantley LC (2007) AKT/PKB signaling navigating downstream. Cell 129:1261-1274

11. Salau, A. O., Jain, S. (2019) Feature extraction: A survey of the types, techniques, and applications. 5th IEEE International Conference on Signal Processing and Communication (ICSC), Noida, India, 158-164. DOI: https://doi.org/10.1109/ICSC45622.2019.8938371

12. Jain S. Communication of signals and responses leading to cell survival/cell death using engineered regulatory networks. PhD Thesis, Jaypee University of Information Technology, Solan, Himachal Pradesh, India, 2012.

13. Weiss, R. (2001) Cellular computation and communications using engineered genetic regulatory networks. PhD Thesis, MIT.

14. Gaudet S., Kevin J. A., John A. G., Emily P.A., Douglas L. A, Peter S. K. (2005) A compendium of signals and responses trigerred by prodeath and prosurvival cytokines. Mol Cell Proteomics, 4(10): 1569-1590. DOl: https://doi. org/10.1074/mcp.M500158-MCP200

15. Jain, S., Chauhan, D. S. (2019) Instance-based learning of marker proteins of carcinoma cells for cell death/survival. Computer Methods in Biomechanics and Biomedical Engineering: Imaging \& Visualization. DOI: https://doi.org/10. 1080/21681163.2019.1692236

\section{Publisher's Note}

Springer Nature remains neutral with regard to jurisdictional claims in published maps and institutional affiliations.

\section{Submit your manuscript to a SpringerOpen ${ }^{\circ}$ journal and benefit from:}

- Convenient online submission

- Rigorous peer review

- Open access: articles freely available online

- High visibility within the field

- Retaining the copyright to your article

Submit your next manuscript at $\boldsymbol{\nabla}$ springeropen.com 